Quim. Nova, Vol. 33, No. 4, 834-840, 2010

\title{
EFEITOS DA ADIÇÃO DE ÁTOMOS DE Mn NA REDE DO GaN VIA MÉTODOS DE ESTRUTURA ELETRÔNICA
}

\author{
Melânia Cristina Mazini e Julio Ricardo Sambrano* \\ Departamento de Matemática, Universidade Estadual Paulista, Av. Eng. Luiz E. C. Coube, s/n, 17033-360 Bauru - SP, Brasil \\ Alberto Adriano Cavalheiro \\ Universidade Estadual de Mato Grosso do Sul, 79950-000 Navirai - MS, Brasil \\ Douglas Marcel Gonçalves Leite e José Humberto Dias da Silva \\ Departamento de Física, Universidade Estadual Paulista, Av. Eng. Luiz E. C. Coube, s/n, 17033-360 Bauru - SP, Brasil
}

Recebido em 6/5/09; aceito em 9/11/09; publicado na web em 10/3/10

\begin{abstract}
INDIRECT EFFECTS OF THE Mn INCORPORATION ON THE ELECTRONIC STRUCTURE OF NANOCRYSTALLINE GaN. A computational method to simulate the changes in the electronic structure of $\mathrm{Ga}_{1-x} \mathrm{Mn}_{x} \mathrm{~N}$ was performed in order to improve the understanding of the indirect contribution of $\mathrm{Mn}$ atoms. This periodic quantum-mechanical method is based on density functional theory at B3LYP level. The electronic structures are compared with experimental data of the absorption edge of the GaMnN. It was observed that the indirect influence of $\mathrm{Mn}$ through the structural parameters can account for the main part of the band gap variation for materials in the diluted regime $(\mathrm{x}<0.08)$, and is still significant for higher compositions $(\mathrm{x} \sim 0.18)$.
\end{abstract}

Keywords: GaN; diluted magnetic semiconductor (DMS); DFT

\section{INTRODUÇÃO}

Estudos recentes mostraram que materiais como GaAs, InAs e $\mathrm{GaN}$, dopados com quantidades de $\mathrm{Mn}, \mathrm{Fe}, \mathrm{Ni}, \mathrm{Co},{ }^{1-8}$ da ordem de $5 \%$ apresentam, além das propriedades semicondutoras, propriedades ferromagnéticas. ${ }^{5,9}$ As propriedades magnéticas destes materiais estão associadas ao fato de que, nos metais de transição citados, os níveis $d$ estão incompletos e apresentam geralmente um valor elevado de momento magnético. Isto confere aos materiais propriedades de semicondutor magnético diluído (DMS).

Os DMSs são potenciais candidatos para utilização em spintrônica, tecnologia na qual os benefícios da carga e do spin do elétron se unem em um único dispositivo, ${ }^{7}$ por possibilitarem a injeção de correntes elétricas com alto grau de polarização de spin entre um metal e um semicondutor que é observada em junções metal semicondutor tradicional. ${ }^{3,10-12}$ Os dispositivos spintrônicos baseados em DMSs apresentam potencial para promover a integração entre comunicação, memória e processamento em um único dispositivo proporcionando ganho extraordinário na velocidade de processamento de dados. ${ }^{3,4,7,13}$ Outro benefício dos dispositivos spintrônicos reside na grande economia de energia, quando comparada aos dispositivos tradicionais. ${ }^{5}$

Entre os DMSs conhecidos o GaN dopado com Mn é um dos mais promissores candidatos a utilização em dispositivos spintrônicos, pois este material pode apresentar uma temperatura de Curie (Tc) acima da temperatura ambiente (Tc $~ 400 \mathrm{~K}$ ). Em particular, materiais como $\mathrm{Ga}_{1-\mathrm{x}} \mathrm{Mn}_{\mathrm{x}}$ As e $\mathrm{In}_{1-\mathrm{x}} \mathrm{Mn}_{\mathrm{x}}$ As apresentam desvantagem de suas temperaturas de Curie encontrarem-se abaixo da temperatura ambiente, Tc $\sim 100 \mathrm{~K} \mathrm{e} \mathrm{Tc} \sim 50 \mathrm{~K}$, respectivamente, tornando-os menos convenientes para uso prático. ${ }^{5,6,14}$ Outros materiais que também apresentaram propriedades ferromagnéticas à temperatura ambiente são o $\mathrm{TiO}_{2}$ e $\mathrm{ZnO}$, dopados com $\mathrm{Mn}$, Fe e Co. ${ }^{15-18}$

Para crescer filmes de $\mathrm{Ga}_{1-\mathrm{x}} \mathrm{Mn}_{\mathrm{x}} \mathrm{N}(\mathrm{GaMnN})$ encontramos técnicas como a Epitaxia por Feixe Molecular (MBE), a qual tem como principal característica o controle do crescimento dos filmes a nível de camadas atômicas, podendo produzir filmes monocristalinos com baixa densidade de defeitos. No entanto, para conseguir certas

\footnotetext{
*e-mail: sambrano@fc.unesp.br
}

concentrações de Mn sem que ocorra segregação desse elemento faz-se necessário o uso de temperaturas mais baixas que as ideais para a preparação do $\mathrm{GaN},{ }^{14}$ de maneira que os materiais podem apresentar grandes quantidades de defeitos..$^{19}$ Outra técnica utilizada para o crescimento de filmes de GaMnN é RF-Magnetron Sputtering reativo, ${ }^{20,21}$ técnica relativamente simples, que apesar de obter filmes policristalinos, possibilita uma melhor incorporação do dopante em concentrações mais elevadas, utilizando baixas temperaturas.

A análise da estrutura eletrônica destes materiais é fundamental para sua aplicação em dispositivos. Neste sentido, a simulação computacional, via métodos de estrutura eletrônica, pode fornecer informações relevantes que podem ser utilizadas como complemento àquelas obtidas por técnicas experimentais, proporcionando uma excelente oportunidade de intercâmbio entre a teoria e o experimento. De forma geral, um resultado experimental é fruto da contribuição e combinação de vários fatores, os quais muitas vezes não podem ser decompostos e estudados separadamente para uma melhor compreensão das propriedades do sistema analisado. A simulação computacional, por outro lado, tem a liberdade de estudar cada aspecto do sistema separadamente e entender qual a contribuição de cada fator nas suas propriedades. Com isso, a simulação computacional aliada a resultados experimentais formam, juntos, uma importante ferramenta para a compreensão das características de sistemas complexos, como é o caso das propriedades eletrônicas e estruturais de semicondutores magnéticos diluídos (DMS).

O objetivo deste trabalho foi analisar, através de uma combinação entre simulação computacional e dados experimentais, a influência da adição de átomos de Mn nas propriedades eletrônicas e estruturais do $\mathrm{GaN}$ na estrutura da wurtzita. Para tal, pretende-se simular, usando parâmetros de rede obtidos experimentalmente, a incorporação de Mn em diferentes concentrações ( $x=0,02 ; 0,05 ; 0,07 ; 0,08$ e 0,18 ), obtidos de filmes nanocristalinos de GaMnN. Os resultados em função da estrutura de bandas e densidade de estados, em particular serão comparados com valores de band gap determinados através de medidas ópticas, os quais refletem as características do GaMnN.

A complexidade do material analisado não permite fazer um cálculo direto, considerando todas as impurezas e defeitos do material. Desta forma, propõe-se uma metodologia alternativa para caracte- 
rizar os efeitos da incorporação de Mn nas propriedades eletrônicas e estruturais do $\mathrm{GaN}$. Os parâmetros estruturais do $\mathrm{GaMnN}$ foram obtidos por meio dos dados de difração de raio-X. A deformação estrutural induzida pela presença do dopante nos permite analisar de maneira indireta a sua influência global na estrutura eletrônica do material, não sendo necessária a inclusão de átomos de Mn diretamente na simulação.

\section{MÉTODO TEÓRICO E MODELO COMPUTACIONAL}

\section{Teoria do funcional da densidade (DFT)}

O método teórico aqui aplicado tem como base a Teoria do funcional da densidade (DFT), desenvolvida nos anos 1960, por W. Kohn, P. Hohemberg e L. Sham. ${ }^{22,23}$ A DFT demonstra que a densidade eletrônica pode ser usada como uma variável fundamental em sistemas moleculares bem como em fase condensada. ${ }^{24-26} \mathrm{Na}$ metodologia DFT a minimização da energia total utilizando as funções densidade eletrônica em função da posição espacial $\rho=\rho(\vec{r})$ é equivalente ao cálculo das funções de onda do estado fundamental do sistema, inclusive para um sistema de muitas partículas.

Na DFT a energia total é função de $\rho$ e das coordenadas $R$ dos núcleos atômicos: $E=E[\rho, R]$. A energia total pode ser decomposta de forma exata em: $E=T[\rho]+U[\rho]+E_{x c}[\rho]$, onde $T[\rho]$ é a energia cinética, $U[\rho]$ é a energia de Coulomb, e $E_{x c}[\rho]$ é o termo que inclui os fatores de troca e correlação eletrônica. A essência da teoria é a minimização de $E$ em relação às (funções) densidades eletrônicas $\rho$.

A metodologia DFT pode ser aplicada a um conjunto muito diversificado de sistemas atômicos, moleculares, líquidos e sólidos, ${ }^{27-30}$ e é um dos métodos teóricos mais utilizados atualmente. Podem-se encontrar excelentes textos de revisão sobre esta metodologia. ${ }^{31}$

Sob certas condições, a densidade eletrônica total pode ser decomposta em densidades monoeletrônicas, originadas das funções de onda monoeletrônicas: $\rho(\vec{r})=\sum|\psi i(\vec{r})|^{2}$.

Assumindo que a energia total assume um ponto mínimo local com relação à densidade eletrônica total do sistema pode-se chegar às Equações de Kohn-Shan:

$\left[-\frac{1}{2} \nabla^{2}+V_{c}(\vec{r})+\mu_{x c}(\vec{r})\right] \psi_{i}=\varepsilon_{i} \psi_{i}$

onde $-1 / 2 \nabla^{2}$ é o operador energia cinética monoeletrônica, $V_{c}(\vec{r})$ é o operador Coulombiano que inclui todas as interações eletrostáticas e $\mu_{x c}(\vec{r})$ é o termo de troca e correlação. Observa-se que a única diferença entre as Equações de Hartree-Fock (HF) e DFT é o termo $\mu_{x c}(\vec{r})$. A energia de troca e correlação pode ser dividida em: $E_{x c}=E_{x}+E_{c}$.

No desenvolvimento da DFT, o primeiro funcional de troca utilizado foi o LDA (aproximação da densidade local) baseado no estado fundamental do gás homogêneo, onde os elétrons se movimentam em um campo uniforme de cargas positivas. Posteriormente surgiram funcionais que melhoram os valores de energia, ao relacionar uma parte HF e outra DFT, chamados funcionais híbridos. Entre estes funcionais híbridos pode-se ressaltar o funcional de três parâmetros de Becke (B3LYP), ${ }^{32,33}$ o qual será utilizado neste trabalho.

\section{Modelo computacional}

Um modelo computacional é constituído de três unidades básicas: uma estrutura molecular, um conjunto de funções de base e nível da teoria a ser aplicado. Quando uma destas partes é modificada se entende que o modelo computacional é outro e seu resultado seguramente será distinto do primeiro modelo. No momento em que se implementa um modelo computacional seus resultados podem ser comparados a resultados experimentais, quando disponíveis, como parâmetro de precisão do cálculo e este deve ser capaz de predizer ou estimar propriedades muitas vezes difíceis ou até mesmo impossíveis de serem obtidas experimentalmente.

Dentro dos métodos computacionais da Química Quântica que permitem predizer, tanto de modo quantitativo como qualitativo, a estrutura e as propriedades de sistemas em fase condensada, podemse distinguir dois grupos; os sólidos cristalinos e os sólidos amorfos. Este estudo estará restrito aos sólidos do primeiro grupo.

A simetria e rigidez da rede cristalina facilitam a construção de estruturas, que podem ser diferenciadas em duas classes: estruturas finitas, denominadas de aglomerados ou modelos de cluster e estruturas periódicas, que consistem na repetição ordenada de átomos ou conjunto de átomos em uma, duas ou três direções. As unidades que se repetem são idênticas e podem ser obtidas mediante operações de simetria de uma célula unitária, este é o principio básico dos modelos periódicos.

Uma etapa importante no processo de cálculo da estrutura eletrônica é a escolha dos funcionais a serem utilizados. É sabido que alguns funcionais são hábeis para predizer a estrutura, mas subestimam o band gap, e outros funcionais que descrevem bem o band gap muitas vezes superestimam os parâmetros estruturais. Recentemente, Bilc e colaboradores $^{34}$ publicaram um estudo comparativo para uma série de funcionais, com o objetivo de predizer as propriedades eletrônicas e estruturais de óxidos. Verificaram que nenhum dos funcionais anteriormente disponíveis seria capaz de descrever, ao mesmo tempo, todas as propriedades eletrônicas e estruturais de diferentes óxidos ferroelétricos com diferentes estruturas e propuseram um novo conjunto de funcionais que descreve com maior precisão tanto as propriedades eletrônicas e estruturais destes óxidos. Verificamos, em alguns de nossos últimos trabalhos publicados, ${ }^{35-40}$ que os funcionais híbridos, tal como o B3LYP, têm apresentado bons resultados com relação à predição do band gap e, também, com relação às propriedades estruturais de óxidos metálicos.

Para os cálculos de estrutura eletrônica do GaN e GaMnN utilizamos o programa CRYSTAL03, ${ }^{41}$ aplicando a teoria do funcional da densidade a modelos periódicos, com o funcional híbrido B3LYP ${ }^{32,33}$ associado ao conjunto de funções de base $86-4111 \mathrm{~d} 41 \mathrm{G}^{42,43}$ e $6-21 \mathrm{G}^{* 44}$ para os átomos de $\mathrm{Ga}$ e N, respectivamente. O programa CRYSTAL é baseado na aproximação LCAO (combinação linear dos orbitais atômicos), onde cada orbital se expressa como uma combinação linear de funções de Bloch. ${ }^{41} \mathrm{O}$ programa gráfico XcrysDen ${ }^{45}$ foi utilizado na análise e obtenção das figuras e resultados de estrutura de bandas.

Em nossos estudos foram testados outros funcionais, tais como LDA, GGA, PBE, mas o funcional híbrido foi o que apresentou resultados mais próximos do experimental.

O sistema físico em que estamos interessados, constituído por filmes nanocristalinos de GaMnN, apresenta alto grau de complexidade. Primeiramente, os filmes apresentam cristalitos de dimensões nanométricas $(\sim 30 \mathrm{~nm})$ cercados por regiões de contorno de grãos. Nos contornos de grão as distorções estruturais e a densidade de defeitos são elevadas. Existem também regiões intersticiais, geralmente amorfas, com alta densidade de defeitos. Até mesmo no interior dos cristalitos há um alto grau de desordem, a qual é causada por defeitos e pela presença, dos íons Mn substitucionais ao Ga, em sítios aleatórios.

Mesmo com os notáveis avanços na capacidade computacional das últimas décadas, os sistemas computacionais disponíveis no momento não permitem acesso a tal grau de complexidade. Por isso adotamos aqui um modelo alternativo simples, mas que vem complementar determinados aspectos dos resultados existentes na literatura ${ }^{46,47}$ para o GaMnN monocristalino.

O procedimento utilizado aqui consiste basicamente em calcular a estrutura eletrônica com parâmetros de rede experimentais obtidos do 
GaMnN e analisar os efeitos causados nas propriedades eletrônicas, comparando-os com os resultados experimentais. Isto é, a simulação da incorporação do elemento Mn é considerada somente pelo efeito da perturbação que este produziu na rede cristalina. Desta forma pôde-se analisar a influência indireta do Mn na estrutura eletrônica do GaN. Esta é uma metodologia alternativa ${ }^{48}$ para caracterizar os efeitos da incorporação de $\mathrm{Mn}$ nas propriedades eletrônicas e estruturais do GaN.

Os dados experimentais revelam que a incorporação do Mn introduz um aumento do parâmetro de rede dos cristalitos. ${ }^{20,21}$ Este aumento observado do parâmetro de rede dos cristalitos é aproximadamente linear em relação ao conteúdo de Mn incorporado.

Não estão presentes importantes efeitos da orientação de spin, causados principalmente pela introdução dos orbitais $3 \mathrm{~d}$ semipreenchidos dos átomos de Mn. Apesar disto, observamos efeitos que se aproximam dos resultados experimentais e que fornecem indicações interessantes sobre as alterações na estrutura eletrônica do GaMnN.

Os parâmetros de rede experimentais utilizados nos cálculos do $\mathrm{GaN}$ e GaMnN foram determinados através de refinamento Rietveld. ${ }^{21}$

A fase termodinamicamente mais estável do GaN é a fase hexagonal do tipo wurtzita (B4), ${ }^{49}$ grupo espacial $\mathrm{P}_{3} \mathrm{~cm},{ }^{50}$ no qual cada átomo de $\mathrm{Ga}$ se encontra no centro de um tetraedro distorcido coordenado a quatro outros nitrogênios vizinhos. Esta estrutura possui dois parâmetros de rede externos $\boldsymbol{a}$ (plano basal) e $\boldsymbol{c}$ (plano uniaxial) e uma coordenada interna $\boldsymbol{u}$, que descreve as posições relativas do ânion e do cátion ao longo do eixo $z$, Figura 1.

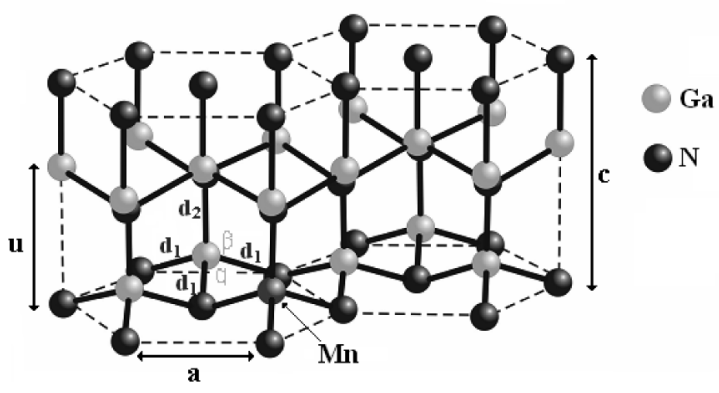

Figura 1. Célula unitária da estrutura do tipo Wurtzita do GaN com um átomo de Mn em um sítio substitucional ao $\mathrm{Ga}$

Para efeito de comparação elaborou-se um modelo teórico $\left(\mathbf{G a N}_{\text {Teor }}\right)$, minimizando-se a energia da célula unitária com relação aos parâmetros de rede e coordenada interna $\boldsymbol{u}$.

\section{RESULTADOS E DISCUSSÃO}

A Tabela 1 apresenta os parâmetros de rede, coordenada interna, distâncias de ligação $\mathrm{Ga}-\mathrm{N}$ e ângulos de ligação $\mathrm{N}-\mathrm{Ga}-\mathrm{N}$ no tetraedro $\left[\mathrm{GaN}_{4}\right]$ (Figura 1) obtidos teorica e experimentalmente pelo refinamento de Rietveld, bem como dados teóricos disponíveis da literatura.

A otimização da célula unitária do GaN puro, $\mathbf{G a N}_{\text {Tẹor., }}$ teve como resultados os seguintes valores: $\boldsymbol{a}=3,193 \AA, \boldsymbol{c}=5,174 \AA$ e $\boldsymbol{u}=0,379$ Å. O processo de otimização apresentou uma boa concordância quando os parâmetros de rede são comparados aos dados experimentais existentes para o $\mathrm{GaN}$ e também aos dados teóricos disponíveis na literatura..$^{51-53}$

Quando os resultados são comparados com os obtidos para o GaN nanocristalino $(\mathrm{x}=0)$ preparado por sputtering verificamos que há uma diferença de parâmetro de rede e valor calculado do band gap, tanto em relação aos dados experimentais obtidos para o $\mathrm{GaN},{ }^{20,21}$ como para os resultados da simulação. Quando consideramos amostras com Mn incorporado, o refinamento de Rietveld mostra que os parâmetros de rede sofrem um aumento em função da adição de Mn na rede, porém o fator $\mathbf{c} / \boldsymbol{a}$ permanece praticamente inalterado, com exceção das proporções $\mathrm{x}=0,02$ e 0,08 . Observa-se também um aumento do volume da célula unitária, de 46,263 $\AA^{3}(\mathrm{x}=0)$ até $47,472 \AA^{3}(\mathrm{x}=0,18)$, equivalente a uma variação de $2,61 \%$, naturalmente associada ao maior tamanho do Mn quando comparado ao átomo de $\mathrm{Ga}$.

Cabe também observar que o aumento do volume da célula unitária em função da inserção de Mn é responsável por uma maior distorção da estrutura cristalina. De fato, para o modelo do $\mathbf{G a N}_{\text {Teor }}$ verifica-se que as distâncias de ligações $\mathrm{Ga}-\mathrm{N}$ na base do tetraedro são de $1,947 \AA$, enquanto que a distância de ligação $\mathrm{Ga}-\mathrm{N}$ na direção $c$ é de $1,962 \AA$ A. Os ângulos de ligação $\mathrm{N}-\mathrm{Ga}-\mathrm{N}$ na base do tetraedro são de $110,20^{\circ}$ e na direção $c$ é de $108,73^{\circ}$ (Tabela 1 ).

Quando consideramos os modelos baseados no refinamento de Rietvield se observa que até $\mathrm{o}$ valor de $\mathrm{x}=0,05$ de $\mathrm{Mn}$ as distâncias de ligação $\mathrm{Ga}-\mathrm{N}$, na base do tetraedro, têm um ínfimo acréscimo de aproximadamente $0,01 \AA$. Neste mesmo intervalo, $x \leq 0,05$, os ângulos de ligação na base do tetraedro tendem a diminuir de $112,70^{\circ}$ $(\mathrm{x}=0)$ para $112,32^{\circ},(\mathrm{x}=0,05)$. Pode-se concluir que há uma expansão isotrópica da rede para pequenas quantidades de $\mathrm{Mn}$.

Para quantidades acima de $\mathrm{x}=0,07$ de $\mathrm{Mn}$ as distâncias de ligação $\mathrm{Ga}-\mathrm{N}$ na base do tetraedro sofrem um decréscimo, iniciando em $1,933 \AA(\mathrm{x}=0,07)$ até $1,887 \AA(\mathrm{x}=0,18)$, por outro lado a distância de ligação $\mathrm{Ga}-\mathrm{N}$ na direção $c$ sofre acréscimo de $0,267 \AA$. Esta

Tabela 1. Parâmetros de rede $\boldsymbol{a}$ e $\boldsymbol{c}$, coordenada interna $\boldsymbol{u}$; $\boldsymbol{c} \boldsymbol{a}(\AA)$; distância de ligação $(\AA)$, Ga $-\mathrm{N}$ na base do tetraedro $\left[\mathrm{GaN}_{4}\right]\left(\mathrm{d}_{1}\right), \mathrm{Ga}-\mathrm{N}$ na direção $c\left(\mathrm{~d}_{2}\right)$, ângulo de ligação (graus), $\mathrm{N}-\mathrm{Ga}-\mathrm{N}$ na base do tetraedro $(\alpha), \mathrm{N}-\mathrm{Ga}-\mathrm{N}$ na direção $c(\beta)$, razão $\mathrm{d}_{1} / \mathrm{d}_{2}$ e volume, $\boldsymbol{V}\left(\AA^{3}\right)$

\begin{tabular}{lccccccccccc}
\hline & $\mathrm{Ga}_{1-\mathrm{x}} \mathrm{Mn}_{\mathrm{x}} \mathrm{N}$ & $a$ & $c$ & $u$ & $c / a$ & $\mathrm{~d}_{1}$ & $\mathrm{~d}_{2}$ & $\alpha^{*}$ & $\beta^{*}$ & $\mathrm{~d}_{1} / \mathrm{d}_{2}$ & $V$ \\
\hline Filmes por & $\mathrm{x}=0,00$ & 3,2039 & 5,2041 & 0,398 & 1,624 & 1,924 & 2,071 & 112,70 & 106,01 & 0,929 & 46,263 \\
RF-Magnetron & $\mathrm{x}=0,02$ & 3,2070 & 5,2042 & 0,395 & 1,623 & 1,931 & 2,056 & 112,32 & 106,44 & 0,939 & 46,343 \\
Sputtering & $\mathrm{x}=0,05$ & 3,2111 & 5,2137 & 0,395 & 1,624 & 1,933 & 2,059 & 112,32 & 106,45 & 0,939 & 46,557 \\
Reativo $^{21}$ & $\mathrm{x}=0,07$ & 3,2161 & 5,2242 & 0,397 & 1,624 & 1,933 & 2,074 & 112,57 & 106,16 & 0,932 & 46,796 \\
& $\mathrm{x}=0,08$ & 3,2219 & 5,222 & 0,404 & 1,621 & 1,927 & 2,110 & 113,48 & 105,08 & 0,913 & 46,945 \\
& $\mathrm{x}=0,18$ & 3,2316 & 5,2489 & 0,446 & 1,624 & 1,887 & 2,341 & 117,79 & 98,64 & 0,806 & 47,472 \\
& GaN $_{\text {Teor. }}^{*}$ & 3,193 & 5,174 & 0,379 & 1,620 & 1,947 & 1,962 & 110,20 & 108,73 & 0,992 & 45,685 \\
& PPPW/LDA $^{51}$ & 3,18 & 5,183 & 0,3765 & 1,63 & & & & \\
& PPPW/GGA $^{51}$ & 3,25 & 5,298 & 0,3765 & 1,63 & & & & & \\
& PPPW/LDA & 3,193 & 5,218 & 0,376 & 1,634 & & & & & \\
& FP-LAPW/GGA & 3,18 & 5,166 & 0,376 & 1,625 & & & & & \\
& GaNexp $^{52}$ & 3,192 & 5,166 & 0,375 & 1,624 & & & & & \\
GaNexp $^{53}$ & 3,18 & 5,166 & 0,375 & & & & & &
\end{tabular}

* este trabalho. 
expansão pode ser explicada pelo fato de que a inserção de átomos de $\mathrm{Mn}$ na rede do $\mathrm{GaN}$ exige um maior espaço para sua estabilização. Observa-se também que os ângulos de ligação $\mathrm{N}-\mathrm{Ga}-\mathrm{N}$, na base do tetraedro, sofrem um acréscimo de aproximadamente $5 \%$ para a proporção de $\mathrm{x}=0,18$ de $\mathrm{Mn}$.

Analisando ainda os aspectos locais, verifica-se que da população de recobrimento (Tabela 2), que a ligação $\mathrm{Ga}-\mathrm{N}$ da base do tetraedro passa a ter um caráter mais covalente, enquanto que a ligação $\mathrm{Ga}-\mathrm{N}$ na direção $c$ passa a ter um caráter parcialmente iônico.

Tabela 2. Band gap $(\mathrm{eV})$ experimental e teórico, energia de Fermi, $\mathrm{E}_{f}(\mathrm{eV}) \mathrm{e}$ população de recobrimento, $\mathrm{d}_{1}$ e $\mathrm{d}_{2}(\mathrm{~m}|\mathrm{e}|)$

\begin{tabular}{lcccccc}
\hline & $\mathrm{Ga}_{1-\mathrm{x}} \mathrm{Mn}_{\mathrm{x}} \mathrm{N}$ & gap $_{\text {exp }}$ & gap & $\mathbf{E}_{f}^{*}$ & $\mathbf{d}_{1}^{*}$ & $\mathbf{d}_{2}^{*}$ \\
\hline Filmes por & $\mathrm{x}=0,00$ & 3,23 & $3,60 *$ & 0,15 & 232 & 193 \\
RF-Magnetron & $\mathrm{x}=0,02$ & 3,17 & $3,60^{*}$ & 0,11 & 231 & 197 \\
Sputtering & $\mathrm{x}=0,05$ & 3,10 & $3,57 *$ & 0,09 & 231 & 197 \\
Reativo $^{21}$ & $\mathrm{x}=0,07$ & 2,91 & $3,49 *$ & 0,09 & 232 & 194 \\
& $\mathrm{x}=0,08$ & 2,88 & $3,42^{*}$ & 0,14 & 234 & 183 \\
& $\mathrm{x}=0,18$ & 2,07 & $3,32 *$ & 0,22 & 246 & 118 \\
& GaN $_{\text {Teor. }}{ }^{2}$ & & $3,87 *$ & 0,00 & 226 & 220 \\
& GaNexp $^{52}$ & 3,44 & & & & \\
& GaNexp $^{53}$ & 3,42 & & & & \\
& PPPW/LDA $^{52}$ & & 1,76 & & & \\
& FP-LAPW/GGA & & 1,992 & & & \\
\hline
\end{tabular}

* este trabalho.

Além dos aspectos locais da estrutura e distribuição de cargas, o modelo aplicado aqui permite a análise também de aspectos de longo alcance relacionados com a estrutura de bandas do material.

Na Tabela 2 observam-se os valores dos band gaps obtidos experimental e teoricamente e energia de Fermi teórica. $\mathrm{O}$ band gap experimental é estimado a partir das bordas de absorção óptica, obtidas por meio de medidas de transmitância. ${ }^{54}$

As Figuras 2a, b e c ilustram as estruturas de bandas para o modelo $\mathrm{GaN}_{\text {teor }}, \mathrm{GaN}$ puro $(\mathrm{x}=0)$ e dopado com $\mathrm{Mn}(\mathrm{x}=0,18)$, respectivamente. A escolha do caminho na primeira zona de Brillouin deve-se ao fato de que este representa a região que contém todos os pontos equivalentes no espaço recíproco com relação ao grupo de simetria utilizado. ${ }^{55}$

Realizou-se a análise da estrutura de bandas para as diferentes proporções de Mn, verificando-se tendências similares no comportamento da estrutura eletrônica das diferentes concentrações; sendo assim, apresentamos a estrutura de bandas apenas para os sistemas $\mathrm{Ga}_{1-\mathrm{x}} \mathrm{Mn}_{\mathrm{x}} \mathrm{N}$ com $\mathrm{x}=0$ e $\mathrm{x}=0,18$.

As pequenas diferenças estruturais levam a uma perturbação dos orbitais moleculares, permitindo caracterizar a desordem no material, este é um exemplo de como a teoria pode auxiliar fornecendo subsídios para os experimentalistas.

Observa-se que o incremento de Mn na rede acarreta em um decréscimo do band gap teórico (DFT), partindo de 3,60 eV do modelo puro para 3,32 eV do modelo dopado com $\mathrm{x}=0,18 \mathrm{de} \mathrm{Mn}$, em acordo com a tendência observada para o band gap medido experimentalmente, que decresce de 3,23 eV, em $\mathrm{x}=0$, para 2,07 eV, em $\mathrm{x}=0,18$. Todos os modelos apresentam um band gap direto no ponto $\Gamma$.

Com o acréscimo de átomos de Mn o band gap teórico apresenta algumas discrepâncias em relação ao band gap experimental (Tabela 2). Neste ponto, ressalta-se que muitas vezes os dados obtidos por meio da simulação não devem ser analisados fazendo-se uma correspondência direta com a magnitude dos resultados experimentais e sim com relação à tendência e comportamentos obtidos. As estimativas experimentais e teóricas apontam para um decréscimo do band gap, mesmo que o decréscimo experimental seja mais acentuado que o apontado teoricamente.

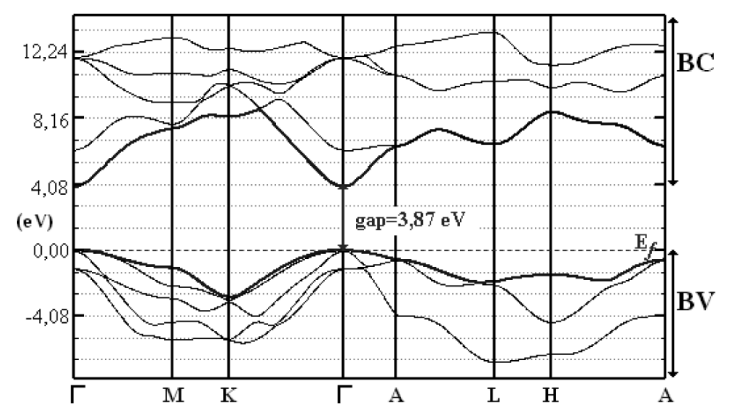

(a)

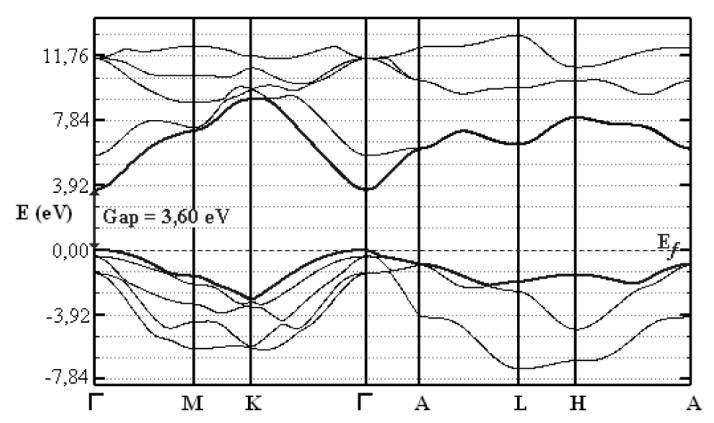

(b)

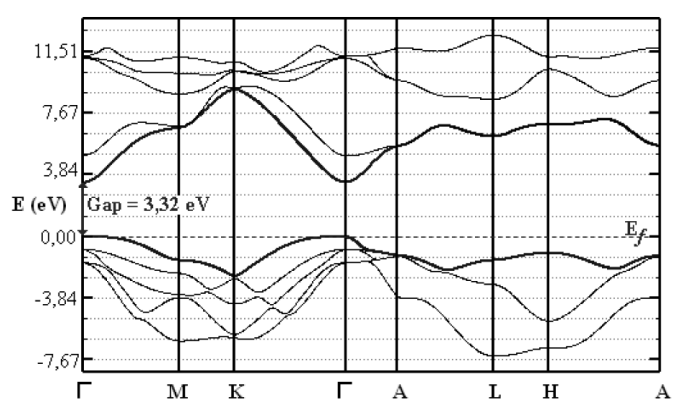

(c)

Figura 2. Estrutura de bandas para a) $\mathbf{G a N}_{\text {Teor. }}$; b) $G a_{1-x} \operatorname{Mn} n_{x} N \operatorname{com} x=0 e$ c) $G a_{1-x} M n_{x} N \operatorname{com} x=0,18$

Também se deve levar em consideração que os valores obtidos teoricamente consideram o GaN como um cristal perfeito, sem nenhum tipo de defeito; esta aproximação pode levar a desvios em relação aos obtidos experimentalmente. Numa amostra do material, devemos considerar que algumas variáveis podem interferir no valor obtido experimentalmente como, por exemplo, a policristalinidade, as técnicas e condições de preparo, a presença de defeitos (vacâncias e impurezas), a porosidade e o tamanho do contorno de grão.

Parte deste decréscimo se deve ao aumento dos parâmetros de rede do $\mathrm{Ga}_{1-x} \mathrm{Mn}_{\mathrm{x}} \mathrm{N}$ devido à incorporação de $\mathrm{Mn}$. Outro fator a considerar na variação do band gap experimental é o fato do átomo de Mn possuir características eletrônicas e químicas diferentes do átomo de $\mathrm{Ga}$.

Existem indícios que o incremento de átomos de manganês levado a proporções próximas de $60 \%$ deve levar a um decréscimo acentuado do band gap, favorecido pelas vacâncias de $\mathrm{Ga},{ }^{56}$ resultando em uma transição de fase da estrutura wurtzita para uma estrutura do tipo blenda. Modelos teóricos e experimentais confirmam que o $\mathrm{MnN}$ existe somente na estrutura do tipo blenda e rocksalt, com um 
band gap de 0,2-0,3 eV. Miao e colaboradores realizaram um estudo teórico onde determinam que a estrutura da blenda é ligeiramente mais estável que a estrutura rocksalt,${ }^{57}$ ao contrário do determinado pelo trabalho experimental do grupo de Suzuki ${ }^{58}$ e Yang. ${ }^{59}$

$\mathrm{Na}$ Figura $2 \mathrm{~b}$, correspondente à estrutura do $\mathrm{GaN}$ nanocristalino sem dopagem, pode-se observar uma leve separação entre as subbandas, na direção $\Gamma$-M. Este efeito deve-se à modificação da simetria local em relação ao cristal. Esta modificação é equivalente no cristal a um stress biaxial em plano perpendicular ao eixo c..$^{55,60,61}$ As mudanças mais fortes nas proximidades do ponto $\Gamma$ ocorrem para a simulação da amostra com maior dopagem ( $\mathrm{x}=0,18)$, da Figura 2c. Esta mudança pode ser associada a um stress uniaxial, na direção do eixo c, produzido pelo aumento do parâmetro $c$ da rede do GaN com a inclusão do Mn.

Notáveis também são as mudanças introduzidas pela nanocristalinidade no intervalo M-K. Por situar-se perpendicular ao eixo $\mathrm{kz}$ no espaço recíproco, o intervalo $\mathrm{M}-\mathrm{K}$ reflete bem as principais mudanças introduzidas pela variação de parâmetro de rede e distância de ligação na direção do eixo $c$ da wurtzita, as quais são os principais efeitos observados, devidos à introdução do Mn. Observa-se também na Figura 2c uma interação no ponto $\Gamma$ com a banda de condução, ou seja, além das modificações observadas na banda de valência, ainda uma mudança na banda de condução pode ser observada, em associação com a presença do $\mathrm{Mn}$.

Os resultados mostram que o aumento do caráter iônico das ligações é compatível com o aumento do parâmetro $c$ da rede hexagonal, o qual pode explicar o efeito semelhante ao de tensão uniaxial na estrutura de bandas.

Em relação aos efeitos de longo alcance, quando o Mn é efetivamente incorporado à estrutura eletrônica do $\mathrm{GaMnN}$ monocristalino (e com o Mn colocado em posições aleatórias no material, o que efetivamente também não é o caso encontrado aqui), há dois regimes: um de baixa diluição no qual os estados do Mn apresentam uma banda isolada no band gap, que é efetivamente determinado pelas bandas do material hospedeiro (como estamos analisando aqui) e outra situação, para altos conteúdos de $\mathrm{Mn}$ nos quais a interação entre as bandas e os estados produzidos pelo Mn é inevitável. Neste caso ocorre uma modificação efetiva nas bandas, passando os estados do Mn a fazer um mix com as bandas e provocar uma redução efetiva do band gap de natureza diferente daquela produzida pelas bandas. Esta ausência efetiva dos estados do Mn deve ser a causa da menor diminuição de band gap observada em nossas simulações em relação aos valores medidos experimentalmente. Aqui, portanto a estrutura eletrônica simulada em relação aos parâmetros de rede mostra apenas uma parte do efeito: as contribuições indiretas para a redução do band gap causada pelo aumento dos parâmetros de rede do GaMnN produzidos pela incorporação de Mn. Nossa contribuição indica que a presença efetiva do Mn é importante para a determinação da variação do band gap no GaMnN nanocristalino, mas que este não é o único efeito a ser considerado para esta variação, pois a variação indireta pelo parâmetro de rede também é significativa.

A Figura 3 ilustra a densidade de estados para o modelo de referência $\mathbf{G a N}_{\text {teor. }}$ e $\mathrm{Ga}_{1-\mathrm{x}} \mathrm{Mn}_{\mathrm{x}} \mathrm{N}, \mathrm{x}=0$ e x $=0,18$, respectivamente. De maneira geral, não existem diferenças significativas quando comparamos a DOS do modelo teórico com os modelos baseados nos resultados de refinamento.

Os diagramas demonstram que para os modelos com $\mathrm{x}=0$ e $\mathrm{x}=$ 0,18 a maior contribuição para a região do topo da banda de valência deve-se aos orbitais $2 \mathrm{p}$ dos átomos de $\mathrm{N}$, com uma maior influência dos orbitais $2 p_{z}$. Na região mais interna da BV observa-se uma relevante participação dos orbitais $3 \mathrm{~d}$ dos átomos de Ga com uma pequena contribuição dos orbitais 2 s e 2 p dos átomos de N. A maior contribuição dos orbitais $\mathrm{p}_{z}$ é relacionada com o fato de que a inserção de átomos de Mn leva a uma deformação preferencial na direção $\boldsymbol{c}$.

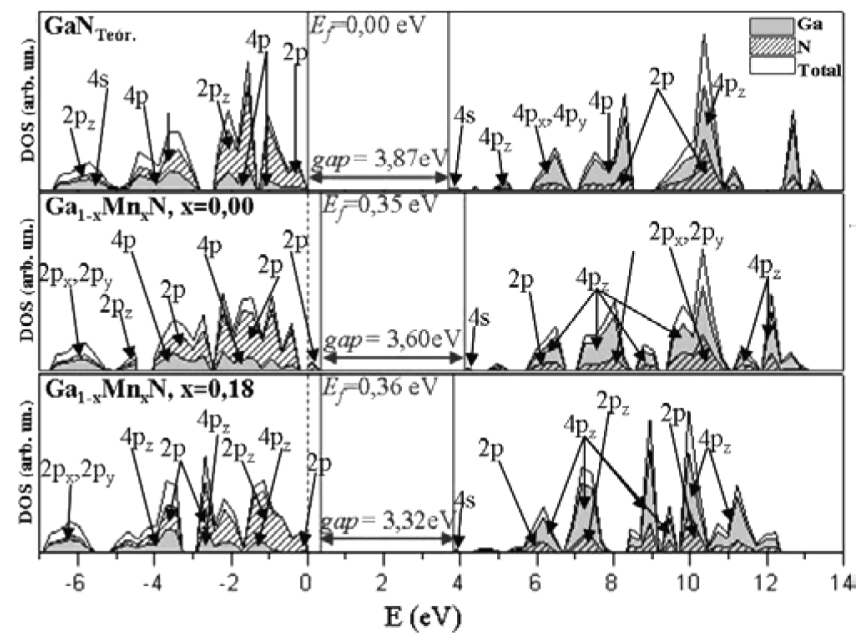

Figura 3. DOS projetado sobre os átomos de Ga e $N$ para $G a N_{\text {Teor. }} ; G a_{1-x} M n_{x} N$ $\operatorname{com} x=0$ e Ga $a_{1-x} n_{x} N \operatorname{com} x=0,18$

A presença do $\mathrm{Mn}^{46,47}$ deve produzir estados localizados no interior do band gap, associados aos níveis Mn-3d semi-preenchidos. A simetria local tetraédrica indica que os orbitais $d$ possuem energias menores que os orbitais $t$, por efeito do campo cristalino. Além disso, a ocupação com spin-up e spin-down produz também uma diferença de energia nestes níveis localizados. Desta maneira podemos esperar que no material real o band gap seja "populado" por estes níveis contribuindo com a redução do seu valor efetivo. Esta contribuição direta do Mn também é somada à interação de seus orbitais s e p das suas ligações de valência, alterando também significativamente estas bandas.

Embora os valores obtidos de band gap sejam significativamente diferentes dos valores experimentais, observamos que no limite diluído $\mathrm{x} \leq 0,08$ a tendência de comportamento em função de $\mathrm{x}$ é semelhante à observada nos dados experimentais. Analisando em termos quantitativos, vemos que para $\mathrm{x}=0,08$, que denominamos de limite de composição diluída, $\Delta_{\text {Teor }}=0,18 \mathrm{eV}$ e $\Delta_{\operatorname{Exp}}=0,35 \mathrm{eV}$, e $\Delta_{\text {Teor } /} \Delta_{\text {Exp }}=0,51$. A variação teórica do band gap corresponde a $51 \%$ da observada experimentalmente. Situação diferente ocorre para $\mathrm{x}$ $=0,18$, na qual a maior parte da variação do band gap não pode ser explicada pelo modelo aqui utilizado, já que $\Delta_{\text {Teor } /} \Delta_{\text {Exp }}=0,24$, ou seja, apenas $\sim 1 / 4$ da variação pode ser explicada pelo modelo aqui apresentado. Entretanto, o valor de $\mathrm{x}=0,18$ que não corresponde mais ao regime diluído, e certamente a introdução dos estados do Mn introduz uma interação direta destes com os estados de banda, sendo os níveis de energia resultantes provenientes desta mistura.

\section{CONCLUSÕES}

As simulações dos parâmetros estruturais e eletrônicos do GaN monocristalino puro com estrutura wurtzita apresentam uma boa concordância com resultados experimentais e teóricos disponíveis na literatura. Em particular a comparação dos valores calculados do band gap, 3,87 eV, com os resultados experimentais, $3,23 \mathrm{eV}^{21} \mathrm{e}$ $\sim 3,4 \mathrm{eV}^{52,53}$, encontra-se dentro do que se pode esperar em modelos periódicos que empregam a DFT.

Em relação aos filmes nanocristalinos não dopados, observa-se que o band gap calculado apresenta uma redução em relação ao valor obtido para o GaN. O decréscimo observado (de 3,87 para 3,60 eV) está em concordância com o decréscimo verificado nos resultados experimentais (de 3,40 para 3,23 eV).

Nos filmes nanocristalinos dopados com Mn, a inserção de Mn na rede leva a um aumento dos parâmetros de rede experimentais. Intro- 
duzindo este efeito nos cálculos da estrutura eletrônica, observa-se que o incremento de átomos de Mn é responsável por uma maior distorção da estrutura cristalina. Os resultados obtidos refletem mudanças na distribuição local de cargas e, também, na estrutura de bandas do material. Em relação aos aspectos locais, a maior contribuição na distorção do tetraedro é devida ao estiramento da ligação $\mathrm{Ga}-\mathrm{N}$ na direção $\boldsymbol{c}$. A ligação $\mathrm{Ga}-\mathrm{N}$ da base do tetraedro passa a ter um caráter mais covalente, enquanto que a ligação $\mathrm{Ga}-\mathrm{N}$ na direção $c$ passa a ter um caráter parcialmente iônico. As modificações de longo alcance observadas na estrutura de bandas em relação ao GaN puro são compatíveis com esta mudança local de caráter da ligação GaN.

Por outro lado, o incremento de Mn na rede acarreta um decréscimo do band gap experimental, cuja tendência é também apresentada nos resultados da simulação. Entretanto o decréscimo experimental é significativamente maior que o verificado na simulação, indicando que os efeitos diretos do Mn também devem ser considerados. Fica claro, entretanto, que a simulação aqui proposta consegue explicar a tendência de variação do band gap, e que os efeitos indiretos do Mn sobre a estrutura eletrônica do material hospedeiro também são significativos e devem ser pensados juntamente com os efeitos diretos produzidos pelas funções de onda do Mn na estrutura eletrônica.

Dentro do regime diluído $\mathrm{x} \leq 0,08$, o modelo aqui proposto, de influência indireta do Mn pode explicar a maior parte das variações observadas no valor do band gap do material, já no regime de alta concentração $(x=0,18)$ apenas cerca de $1 / 4$ da modificação no band gap é produzida pela influência indireta aqui observada, sendo os estados eletrônicos associados ao Mn necessários para a compreensão do que ocorre no material.

Conclui-se que a simulação simples de estrutura eletrônica aqui proposta, associada a parâmetros estruturais experimentais pode fornecer resultados que permitem explicar consistentemente alguns dos mecanismos que influenciam a estrutura eletrônica do $\mathrm{GaN}$ nanocristalino em relação à introdução do Mn.

\section{AGRADECIMENTOS}

Todos os cálculos foram realizados no Laboratório de Simulação Molecular da Unesp de Bauru. Este trabalho foi financiado pela $\mathrm{CNPq}$ e FAPESP. M. Mazini agradece especialmente ao CNPq pelo auxílio concedido. D. M. G. Leite é bolsista de doutoramento da FAPESP.

\section{REFERÊNCIAS}

1. Kilanski, L.; Gorska, M.; Domukhovski, V.; Dobrowolski, W.; Anderson, J. R.; Rotundu, C. R.; Varniavskii, S. A.; Marenkin, S. F.; Acta Phys. Pol., A 2008, 114, 1151.

2. Sadowski, J.; Acta Phys. Pol., A 2008, 114, 1001.

3. Ohno, H.; Science 1998, 281, 951 .

4. Ohno, Y.; Young, D. K.; Beschoten, B.; Matsukura, F.; Ohno, H.; Awschalom, D. D.; Nature 1999, 402, 790.

5. Dietl, T.; Ohno, H.; Matsukura, F.; Cibert, J.; Ferrand, D.; Science 2000 287, 1019

6. Jain, M.; Kronik, L.; Chelikowsky, J. R.; Godlevsky, V. V.; Phys. Rev. B 2001, 64, 245205.

7. Wolf, S. A.; Awschalom, D. D.; Buhrman, R. A.; Daughton, J. M.; von Molnar, S.; Roukes, M. L.; Chtchelkanova, A. Y.; Treger, D. M.; Science 2001, 294, 1488

8. Dietl, T.; Semicond. Sci. Technol. 2002, 17, 377.

9. Overberg, M. E.; Thaler, G. T.; Frazier, R. M.; Abernathy, C. R.; Pearton, S. J.; Rairigh, R.; Kelly, J.; Theodoropoulou, N. A.; Hebard, A. F.; Wilson, R. G.; Zavada, J. M.; J. Appl. Phys. 2003, 93, 7861.

10. Pearton, S. J.; Abernathy, C. R.; Ren, F.; Gallium Nitride Processing for Electronics, Sensors, and Spintronics, Springer Verlag: Londres, 2006.
11. Jonker, B. T.; Proc. IEEE 2003, 91, 727.

12. Schmidt, G.; Ferrand, D.; Molenkamp, L. W.; Filip, A. T.; van Wees, B. J.; Phys. Rev. B 2000, 62, R4790.

13. Awschalom, D. D.; Loss, D.; Samarth, N.; Semiconductor Spintronics and Quantum Computation, Springer Verlag: Berlim, 2002.

14. Thaler, G.; Frazier, R.; Gila, B.; Stapleton, J.; Davidson, M.; Abernathy, C. R.; Pearton, S. J.; Segre, C.; Appl. Phys. Lett. 2004, 84, 1314.

15. Che, P.; Liu, W. B.; Guo, L.; He, L.; Chen, C. P.; J. Magn. Magn. Mater. 2008, 320, 2563.

16. Seo, S. Y.; Kwak, C. H.; Lee, Y. B.; Kim, S. H.; Park, S. H.; Han, S. W.; J. Korean Phys. Soc. 2008, 53, 249.

17. Zhao, Z. Y.; Liu, Q. J.; Zhang, J.; Zhu, Z. Q.; Acta Phys. Sinica 2007, $56,6592$.

18. Malguth, E.; Hoffmann, A.; Phillips, M. R.; Phys. Status Solidi B 2008, 245, 455.

19. Pearton, S. J.; Abernathy, C. R.; Overberg, M. E.; Thaler, G. T.; Norton, D. P.; Theodoropoulou, N.; Hebard, A. F.; Park, Y. D.; Ren, F.; Kim, J.; Boatner, L. A.; J. Appl. Phys. 2003, 93, 1

20. Leite, D. M. G.; da Silva, L. F.; Pereira, A. L. J.; da Silva, J. H. D.; J. Cryst. Growth 2006, 294, 309.

21. da Silva, J. H. D.; Leite, D. M. G.; Tabata, A.; Cavalheiro, A. A.; J. Appl. Phys. 2007, 102, 063526.

22. Hohenberg, P.; Kohn, W.; Phys. Rev. B 1964, 136, B864.

23. Kohn, W.; Sham, L. J.; 1965, 140, 1133.

24. Delley, B.; J. Chem. Phys. 1990, 92, 508

25. Pisani, C.; Ed Quantum Mechanical ab-initio Calculations of the properties of Cristaline Materials, Springer: Heidelberg, 1996.

26. Parr, R. G.; Yang, W.; Density Functional Theory of Atoms and Molecules, Oxford University Press: New York, 1997.

27. Pavao, A. C.; Santos, J. R. S.; Taft, C. A.; Mol. Simul. 2009, 35, 287.

28. Gracia, L.; Polo, V.; Sambrano, J. R.; Andres, J.; J. Phys. Chem. A 2008, 112, 1808.

29. Song, Z. X.; Wang, H. J.; Xing, L. J.; J. Solution Chem. 2009, 38, 1139.

30. Sambrano, J. R.; Longo, V. M.; Longo, E.; Taft, C. A.; J. Mol. Struct. 2007, 813,49

31. Morgon, N. H.; Custodio, R.; Quim. Nova 1995, 18, 44.

32. Lee, C. T.; Yang, W. T.; Parr, R. G.; Phys. Rev. B 1988, 37, 785.

33. Becke, A. D.; J. Chem. Phys. 1993, 98, 5648.

34. Bilc, D. I.; Orlando, R.; Shaltaf, R.; Rignanese, G. M.; Iniguez, J.; Ghosez, P.; Phys. Rev. B 2008, 77, 165107

35. Sambrano, J. R.; Nobrega, G. F.; Taft, C. A.; Andres, J.; Beltran, A.; Surf. Sci. 2005, 580, 71.

36. Beltran, A.; Sambrano, J. R.; Calatayud, M.; Sensato, F. R.; Andres, J.; Surf. Sci. 2001, 490, 116

37. De Lázaro, S. R.; Longo, E.; Beltran, A.; Sambrano, J. R.; Quim. Nova 2005, 28, 10.

38. Marana, N. L.; Longo, V. M.; Longo, E.; Martins, J. B. L.; Sambrano, J. R.; J. Phys. Chem. A 2008, 112, 8958.

39. Beltran, A.; Andres, J.; Sambrano, J. R.; Longo, E.; J. Phys. Chem. A 2008, 112, 8943.

40. Longo, V. M.; Cavalcante, L. S.; Figueiredo, A. T.; Santos, L. P. S.; Longo, E.; Varela, J. A.; Sambrano, J. R.; Paskocimas, C. A.; De Vicente, F. S.; Hernandes, A. C.; Appl. Phys. Lett. 2007, 90, 091906.

41. Dovesi, R.; Saunders, V. R.; Roetti, C.; Zicovich-Wilson, C. M.; Harrison, N. M.; Orlando, R.; Doll, K.; Civalleri, B.; Bush, I.; D’ Arco, P.; Llunell, M.; CRYSTALO3 User's Manual; University of Torino, Italy, 2003.

42. Pandey, R.; Jaffe, J. E.; Harrison, N. M.; J. Phys. Chem. Solids 1994, 55, 1357.

43. Pandey, R.; Causa, M.; Harrison, N. M.; Seel, M.; J. Phys.: Condens. Matter. 1996, 8, 3993.

44. Dovesi, R.; Causa, M.; Orlando, R.; Roetti, C.; Saunders, V. R.; J. Chem. Phys. 1990, 92, 7402 . 
45. Kokalj, A.; J. Mol. Graphics Modell. 1999, 17, 176.

46. Wang, Q.; Sun, Q.; Jena, P.; Phys. Rev. B 2007, 75, 035322.

47. Schulthess, T. C.; Temmerman, W. M.; Szotek, Z.; Svane, A.; Petit, L.; Phys. B 2007, 19, 165207.

48. Sambrano, J. R.; Orhan, E.; Gurgel, M. F. C.; Campos, A. B.; Goes, M. S.; Paiva-Santos, C. O.; Varela, J. A.; Longo, E.; Chem. Phys. Lett. 2005, $402,491$.

49. Vurgaftman, I.; Meyer, J. R.; J. Appl. Phys. 2003, 94, 3675.

50. Ozgur, U.; Alivov, Y. I.; Liu, C.; Teke, A.; Reshchikov, M. A.; Dogan, S.; Avrutin, V.; Cho, S. J.; Morkoc, H.; J. Appl. Phys. 2005, 98, 041301.

51. Saib, S.; Bouarissa, N.; Phys. B 2007, 387, 377.

52. Stampfl, C.; van de Walle, C. G.; Phys. Rev. B 1999, 59, 5521.

53. Ahmed, R.; Akbarzadeh, H.; Fazel e, A.; Physica B 2005, 370, 52.

54. Leite, D. M. G.; da Silva, J. H. D.; J. Phys.: Condens. Matter. 2008, 20, 055001 .
55. Suzuki, M.; Uenoyama, T.; Yanase, A.; Phys. Rev. B 1995, 52, 8132.

56. Chan, J. A.; Liu, J. Z.; Raebiger, H.; Lany, S.; Zunger, A.; Phys Rev B 2008, 78, 184109.

57. Miao, M. S.; Lambrecht, W. R. L.; Phys Rev B 2007, 76, 195209.

58. Suzuki, K.; Yamaguchi, Y.; Kaneko, T.; Yoshida, H.; Obi, Y.; Fujimori, H.; Morita, H.; J. Phys. Soc. Jpn. 2001, 70, 1084.

59. Yang, H. Q.; Al-Brithen, H.; Trifan, E.; Ingram, D. C.; Smith, A. R.; J. Appl. Phys. 2002, 91, 1053.

60. Morkoç, H.; Hamdani, F.; Salvador, A.; Electronic and Optical Properties of III-V Nitrides. In: Semiconductors and Semimetals, Academic Press: San Diego, 1998.

61. Uenoyama, T.; Suzuki, M.; Appl. Phys. Lett. 1995, 67, 17. 